\title{
Ensemble Machine Learning for Estimating Fetal Weight at Varying Gestational Age
}

\author{
Yu Lu, ${ }^{1}$ Xi Zhang, ${ }^{1}$ Xianghua Fu, ${ }^{1}$ Fangxiong Chen, ${ }^{2}$ Kelvin K. L. Wong ${ }^{3, *}$ \\ ${ }^{1}$ Faculty of Arts and Sciences, Shenzhen Technology University, Shenzhen, China \\ ${ }^{2}$ School of Automation, Guangdong University of Technology, Guangzhou, China \\ ${ }^{3}$ Shenzhen Institutes of Advanced Technology, Chinese Academy of Sciences, Shenzhen, China \\ *Corresponding author: kelvin.wong@ siat.ac.cn
}

\begin{abstract}
Obstetric ultrasound examination of physiological parameters has been mainly used to estimate the fetal weight during pregnancy and baby weight before labour to monitor fetal growth and reduce prenatal morbidity and mortality. However, the problem is that ultrasound estimation of fetal weight is subject to populations' difference, strict operating requirements for sonographers, and poor access to ultrasound in low-resource areas. Inaccurate estimations may lead to negative perinatal outcomes. We consider that machine learning can provide an accurate estimation for obstetricians alongside traditional clinical practices, as well as an efficient and effective support tool for pregnant women for self-monitoring. We present a robust methodology using a data set comprising 4,212 intrapartum recordings. The cubic spline function is used to fit the curves of several key characteristics that are extracted from ultrasound reports. A number of simple and powerful machine learning algorithms are trained, and their performance is evaluated with real test data. We also propose a novel evaluation performance index called the intersectionover-union (loU) for our study. The results are encouraging using an ensemble model consisting of Random Forest, XGBoost, and LightGBM algorithms. The experimental results show an loU of 0.64 between predicted range of fetal weight at any gestational age from the ensemble model and that from ultrasound. Comparing with the ultrasound method, the estimation accuracy is improved by $12 \%$, and the mean relative error is reduced by $3 \%$.
\end{abstract}

\section{Introduction}

In obstetrics, both abnormal fetal growth and fetal development are monitored via prenatal testing. However, there are few biomarkers that can be used to accurately predict the fetal growth restrictions (FGR) (Conde-Agudelo et al. 2013), macrosomia, and other abnormalities. Currently, estimated fetal weight (EFW) has became a central indicator for this purpose. It is essential to obtain an accurate estimation of antenatal fetal weight because potential complications may arise from excessive or low fetal birth weight during and after delivery.

The prediction of a fetal birth weight just before the delivery is able to effectively guide obstetricians to choose a more reasonable delivery mode for pregnant women. This

Copyright (C) 2019, Association for the Advancement of Artificial Intelligence (www.aaai.org). All rights reserved. can result in an improved delivery outcome during labour and further reduce complications for mothers and infants after labour (Pressman et al. 2000). Moreover, if the FGR and adverse conditions such as intrauterine hypoxia can be detected in time, it would be greatly beneficial to further reduce the possibility of perinatal mortality of fetuses (Miller and Huppi 2016). Therefore, it is desired that the EFW can be accurate as possible not only at the end of the third trimester but also at any gestational week during pregnancy.

Several methods can be used to predict fetal weight in clinical practice, consisting of abdominal palpation, parturient symphysio-fundal height and abdominal girth measurements, and obstetric ultrasound. Among them, the ultrasound based estimation method is most reliable and objective, and has been used extensively by obstetricians in China. Its principle lies is the use of a class of well-established regression models with multiple parameters standards for fetuses. But, there a number of limitations of such method. First, these regression models were proposed by different clinicians, and are not generally applicable to all populations in the world. As a result, the direct use of such class of models on Chinese population may result in inaccuracy, particularly for excessive or low fetal birth weight. Second, there are also strict requirements for sonographers and specific standards for equipment for performing ultrasound examinations. Factors like deformed fetal head, existence of oligohydramnios and abdominal fat, and poor image quality may all affect the final estimation. Another limitation is that access to obstetric ultrasound remains poor in some most low-resource rural areas and this has significantly affected fetal weight estimation (Wanyonyi and Mutiso 2018).

Other than the traditional methods introduced, machine learning techniques can be applied in this field (Naimi, Platt, and Larkin 2018; Podda, Bacciu, and Micheli 2018; Zhu et al. 2018). The historical data of prenatal examinations can be analysed and the relationship between conceptual entities can be explored through their own training, generalisation, self-organisation, and learning ability. Thus, they are a preferable candidate to make more efficient and reasonable decisions such as fetal weight estimation.

The main contributions of this paper are threefold. First, we establish a dataset consisting of 4,212 clinical records based on the electronic health record of pregnant women from a large hospital in China. Second, we establish a tem- 
poral relationship between the gestational age and the main characteristics of fetal growth on Chinese population. The cubic spline function method was used to fit the relationship between characteristics such as the biparietal diameter (BPD), abdominal circumference (AC), head circumference (HC), and femur length (FL) and the gestational age. In addition, we also consider maternal physiological characteristics, such as the pre-pregnancy body mass index (BMI), uterine height and abdominal circumference. Third, we propose an ensemble learning model, which has obtained better prediction results than any single model. Our model is constructed based on three machine learning algorithms and optimised in parallel via a multi-parameter genetic algorithm, and it has been evaluated on our real dataset and compared to several other methods.

\section{Preprocessing}

\section{Methodology}

The experimental data are obtained from Shenzhen Bao'an Maternity \& Child Healthcare Hospital. A total number of 5,000 samples from 2017 are randomly selected, and no general obstetrics, gynaecology and other general medical histories regarding prenatal care are screened out. It was started before 16 weeks of gestation, as measured by the menstrual date and nutritional health, including the maternal height $(\geq 153 \mathrm{~cm})$, BMI $\left(18.5 \leq B M I<30 \mathrm{~kg} / \mathrm{m}^{2}\right)$, erythroprotein concentration $(\geq 110 \mathrm{~g} / \mathrm{L})$, and whether the pregnant women receive anaemia treatments, or have any special diet recipes. It can effectively reduce the risk factors in the FGR and preterm birth.

At the same time, the distribution of pregnancy tests is not equal, and their types are different during the long observation period of the pregnant women. To ensure sufficient sample distributions, the examination data must be after the 16th week of pregnancy. Effective preprocessing of the data is a key step to improve the accuracy of the prediction model.

Parameters of Predictive Model A hospital identification number for the pregnant women is used as the main index to extract the health records from the beginning of the pregnancy to the delivery for obtaining the birth weight. $\mathcal{Y}$ is defined as the EFW from ultrasound examination, and $\mathcal{X}$ is defined as the set of input parameters for the model. The final dataset $\mathcal{X}$ consists of 14 parameters, consisting of $x_{h}$, $x_{p w}, x_{p}, x_{n}, x_{a}, x_{g}, x_{g g}, x_{f w}, x_{p b}, x_{c b}, x_{B P D}, x_{A C}, x_{H C}$, and $x_{F L}$, and the meaning of each parameter is shown in Table 1.

Feature Standardisation After data preprocessing, 4,212 samples meet the underlying conditions. However, the different physiological parameters have different units and orders of magnitude. To reduce these influences on the prediction results, the data need to be normalised before the model is trained to ensure that each feature is at the same order of magnitude. The normalisation is shown as Equation (1):

$$
y=\frac{2\left(x-x_{\min }\right)}{x_{\max }-x_{\min }}-1
$$

where $x$ represents the current feature value, $x_{\min }$ and $x_{\max }$ represent the minimum and maximum values of the current
Table 1: Symbol definition of different parameters.

\begin{tabular}{lr}
\hline Parameters & Definition \\
\hline$x_{h}$ & Height of a pregnant woman $(\mathrm{cm})$ \\
$x_{p w}$ & Weight of a pregnant woman $(\mathrm{kg})$ \\
$x_{p}$ & Gestational week \\
$x_{n}$ & Number of pregnancy \\
$x_{a}$ & Age of a pregnant woman \\
$x_{g}$ & Weight gain of a pregnant woman $(\mathrm{kg})$ \\
$x_{g g}$ & Fundal height of a pregnant woman \\
$x_{f w}$ & Abdominal circumference \\
$x_{p b}$ & BMI of pre-pregnancy \\
$x_{c b}$ & BMI of current pregnancy \\
$x_{B P D}$ & Fetal biparietal diameter $(\mathrm{cm})$ \\
$x_{A C}$ & Fetal abdominal circumference $(\mathrm{cm})$ \\
$x_{H C}$ & Fetal head circumference $(\mathrm{cm})$ \\
$x_{F L}$ & Fetal femur length $(\mathrm{cm})$ \\
\hline
\end{tabular}

feature, respectively, and $y$ is the normalised feature value. The data range is $[-1,1]$.

Construction of Fitted Function Despite the widespread use of ultrasound technology worldwide, people are concerned about the low rate of detection of fetal developmental abnormalities in routine clinical practice (Ewigman, Crane, and Frigoletto 1993). However, there is a lack of appropriate international standards similar to those used to monitor infant growth (de Onis 2006). In addition, there are some differences in fetal growth characteristics in different regions. Therefore, this study uses the cubic spline function method to fit four characteristics of ultrasound detection.

Specifically, at the interval $[a, b], a=t_{0}<t_{1}<\ldots<$ $t_{n}<t_{(n+1)}=b, f(x)$ is defined as a function of $\left[t_{n}, b\right]$. If $f(x)$ meets the following two conditions: (1) $f(x)$ is a cubic polynomial on each interval of $\left[a, t_{1}\right],\left[t_{1}, t_{2}\right], \ldots,\left[t_{n}, b\right]$ and (2) $f(x)$ and its second derivative are continuous at $t_{i}(i=$ $1,2, \ldots, n)$, then the piecewise polynomial function is called the cubic spline function. The point $t_{i}$ is called the node of the spline function. The cubic spline function can be shown in Equation (2):

$$
f(x)=d_{i}\left(x-t_{i}\right)^{3}+c_{i}\left(x-t_{i}\right)^{2}+b_{i}\left(x-t_{i}\right)+a_{i}
$$

where $t_{i} \leq x \leq t_{i+1}, i=0,1, \ldots, n$. The sum of squared residuals for $t_{i}$ is $\sum\left(y_{i}-g\left(t_{i}\right)\right)^{2}$, and the penalised sum of the squares of the above selection functions is shown in Equation (3):

$$
S(f)=\sum\left(y_{i}-f\left(x_{i}\right)\right)+\gamma \int_{a}^{b}\left(f^{\prime \prime}(x)\right)^{2} d x
$$

For a given smoothing parameter $\gamma$ (whereby $\gamma>0$ ), the estimation function $f(x)$ minimises the values of $S(f)$, which is referred to as a penalty least squares estimate. The smoothing parameter $\gamma$ can be given by $\gamma=\mathcal{C} \mathcal{Q}^{3} / 1000, \mathcal{C}$ is a given constant, and $\mathcal{Q}$ is the interquartile range of the explanatory variable.

\section{Ensemble Machine Learning}

Ensemble methods in machine learning that create multiple models are powerful prediction techniques since they 
can increase the diversity of algorithms and reduce generalisation error to improve the accuracy of the results (Dietterich 2000). This method is divided into stacking, blending and voting. Ensemble methods have two basic elements: one is that the correlation between single models should be as small as possible, and the other is that the performance between single models is not too different. In practice, it is often the case that a single model with a low correlation coefficient and good performance can significantly improve the final prediction result.

Random forest is a supervised learning algorithm (Breiman 2001). The random forest regression algorithm is a combined model, which incorporates a regression decision subtree. According to the principle of ensemble learning, the mean of each decision subtree is taken as the regression prediction result. The random forest is a kind of bagging algorithm, which focuses on reducing the variance. XGBoost (Chen and Guestrin 2006) is a boosting algorithm (Schapire 1990), which focuses on reducing the bias. However, LightGBM (Ke, Meng, and Finley 2017) is a recently proposed algorithm. Therefore, the three classes of algorithms in this paper satisfy the diversity, correlation, and performance requirements. In this study, voting is used to construct an ensemble model, which is shown in Equation (4):

$$
h_{\alpha}(f)=\alpha_{0}+\alpha_{1} f_{1}^{i}+\alpha_{2} f_{2}^{i}+\alpha_{3} f_{3}^{i}
$$

where $\alpha_{1}, \alpha_{2}, \alpha_{3}$ are the weight parameters, $\alpha_{0}$ is a constant, $i$ represents the number of $i$-th samples $i=1,2, \ldots, n$ and $f_{1}, f_{2}, f_{3}$ represent the predicted values of the random forest, XGboost, and LightGBM models, respectively.

\section{Optimisation based on Genetic Algorithm}

According to the above basic model analysis, the parameters that have a large impact on the prediction results of the random forest model, consisting of the following: the maximum number of features is used by a single decision tree $\delta_{\text {max } f}$, the minimum number of leaf nodes $\delta_{\text {min_l }}$, the maximum depth of the decision tree $\delta_{\text {max } d}$, and the minimum number of samples required for the internal node subdivision $\delta_{\text {min_s }}$. For the XGBoost model, the influence factor mainly includes the learning rate $\theta_{\text {eta }}$, the maximum depth of the tree $\theta_{\text {max } d}$, and the minimum leaf node sample weight $\theta_{\text {min_w }}$.

Regarding the LlightGBM model, the influence factors consist of the tree model depth $\gamma_{\text {max } d}$, the minimum number of leaf nodes $\gamma_{\text {min_l }}$, the minimum leaf node weight $\gamma_{\text {min_w }}$, and the learning rate $\gamma_{\text {eta }}$. If a traditional grid search method is used to optimise 15 parameters, then optimisation takes a very long time. The genetic algorithm, as an intelligent evolutionary algorithm, has a strong global search capability. Therefore, this study proposes an ensemble model based on the multi-parameter parallel optimisation of the genetic algorithm. The specific steps are as follows:

1. Data preprocessing: the original data is preprocessed and divided into a training set and a testing set.

2. Initialise parameters of the genetic algorithm such as the population size, crossover probability, and mutation probability.
3. Select the optimisation parameters and interval. According to the above analysis, there are a total of 15 parameters to be optimised: 4 parameters of the random forest model, 3 parameters of the XGBoost model, 4 parameters of the LightGBM model, and 4 parameters of the ensemble model. The optimal interval is determined by chromosome coding.

4. Determine the fitness function. Calculate the average relative error between the predicted value and the true value, so the fitness function is shown in Equation (5):

$$
\begin{aligned}
M A P E= & \frac{1}{n} \sum_{i=1}^{n}\left(\frac{h_{\alpha}\left(f^{i}\right)-y_{i}}{y_{i}}+\left|\frac{f_{1}^{i}-y_{i}}{y_{i}}\right|\right. \\
& \left.+\left|\frac{f_{2}^{i}-y_{i}}{y_{i}}\right|+\left|\frac{f_{3}^{i}-y_{i}}{y_{i}}\right|\right)
\end{aligned}
$$

where $h_{\alpha}\left(f^{i}\right)$ represents the ensemble model predictive value, and $y_{i}$ denotes the true value. Moreover, $f_{1}^{i}, f_{2}^{i}, f_{3}^{i}$ are the output values of the random forest, XGBoost, and LightGBM models, respectively, and $n$ is the number of training sets.

5. Parameter optimisation: First, decode the chromosomes in the population; then calculate the fitness value of each generation of the population, and perform the survival of the fittest. Finally, determine whether the population performance satisfies the maximum number of genetics, and if so, the optimal parameter is output; otherwise, according to the genetic strategy, the selection, crossover and mutation operations are used to obtain the offspring.

6. Result judgement: if the MAPE error requirement is satisfied, then the optimisation is finished. Otherwise, repeat step 4.

7. Input the test sample to obtain the best prediction result. The detailed process is shown in Figure 1.

\section{Performance Evaluation Index}

This paper uses two indices to measure the performance of the ensemble model. The first index is the mean relative error (MRE), which is a measure of the credibility. If $n$ is the number of samples, then the MRE is shown in Equation (6):

$$
M R E=\frac{1}{n} \sum_{i=1}^{n} \frac{\left|y_{\text {true }}-y_{\text {pred }}\right|}{y_{\text {true }}}
$$

where $y_{\text {true }}$ denotes the true label and $y_{\text {pred }}$ denotes the predicted fetal weight.

To better reflect the coincidence between different intervals, this paper introduces a novel concept, originally used in the field of image processing, namely, loU. This method can reflect the coincidence degree of different learning algorithms for predicting the fetal weight interval, and it is shown in Equation (7):

$$
I o U=\frac{f_{\text {scope }}^{1} \cap f_{\text {scope }}^{2}}{f_{\text {scope }}^{1} \cup f_{\text {scope }}^{2}}
$$

where $f_{\text {scope }}^{1}$ represents the fetal weight prediction range of the algorithm model and $f_{\text {scope }}^{2}$ represents the fetal weight range of the ultrasound examination. 

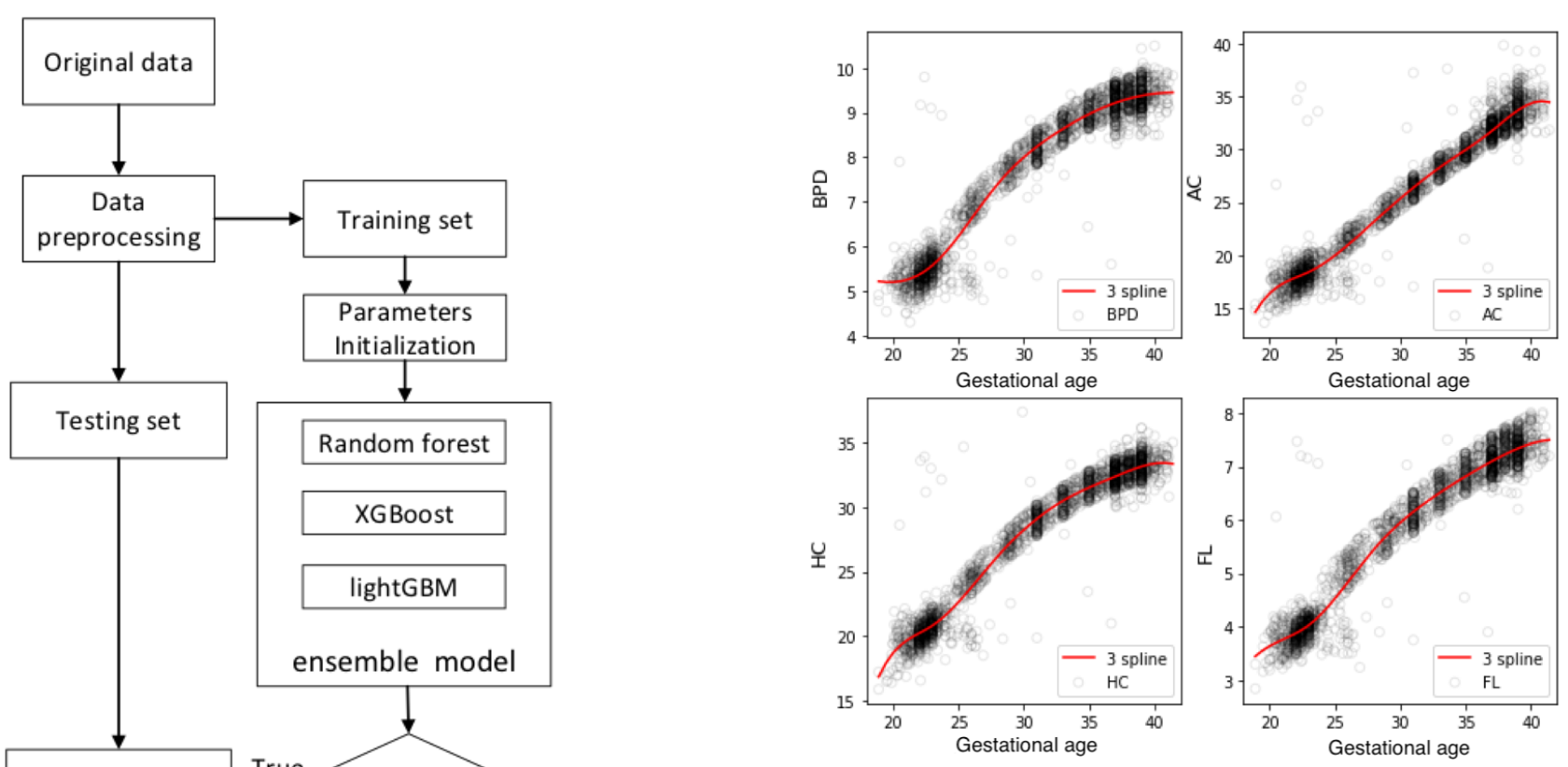

Figure 2: Four feature fitting curves.

0.20 . The minimum $R^{2}$ of the $\mathrm{AC}$ is 0.955 , and the maximum MRE is 0.22 . The minimum $R^{2}$ of the $\mathrm{HC}$ is 0.950 , and the maximum MRE is 0.24 . The minimum $R^{2}$ of the FL is 0.951 , and the maximum MRE is 0.16 . The $R^{2}$ of each index is above 0.95 , and the MRE is within the tolerance; thus, the fitting result is satisfactory.

\section{Evaluation of Prediction}

The random forest, XGboost, LightGBM models and the ensemble model are based on the genetic algorithm. Multiparameter parallel optimisation is used to predict the fetal weight, which is compared with the multi-parameter formula (Hadlock 1990) used in an ultrasonic examination. The experimental results are shown in Figure 3.

As shown in Figure 3, the MRE based on the single machine learning algorithm model is approximately $8 \%$. The MRE of the formula method in (Hadlock 1990) is 14.6\%. The MRE of the ensemble model is approximately $6 \%$. In the absence of ultrasound detection, the fitting function is used to fit the four eigenvalues as shown in Figure 2, and then the integrated model is used to predict the fetal weight range. The loU index is used to prove the effectiveness of the algorithm (see Table 3).

In Table 3, in the absence of an ultrasound examination, the ensemble model, can predict the fetal weight range. Compared with the ultrasonic examination, the loU value is greater than 0.6. To some extent, the fetal weight can be predicted at any gestation according to the maternal characteristic parameters and the fitted four ultrasonic characteristic values. The prediction results of some samples are shown in Figure 4.

In Figure 4, the "0", "1", "2", "3" and "4" values on the horizontal axis represent the ultrasonic examination, the ensemble model in this paper, the XGBoost, the LightGBM, 
Table 2: Fitting results of each percentile curve.

\begin{tabular}{lrrrrrrrrrrrr}
\hline \multirow{2}{*}{ Centile } & \multicolumn{3}{c}{ BPD } & \multicolumn{4}{c}{ AC } & \multicolumn{3}{c}{ HC } & \multicolumn{3}{c}{ FL } \\
\cline { 2 - 14 } & $\gamma$ & MER & $R^{2}$ & $\gamma$ & MER & $R^{2}$ & $\gamma$ & MER & $R^{2}$ & $\gamma$ & MER & $R^{2}$ \\
\hline P95 & 0.3 & 0.16 & 0.954 & 0.4 & 0.22 & 0.958 & 0.4 & 0.20 & 0.956 & 0.4 & 0.16 & 0.951 \\
P75 & 0.2 & 0.12 & 0.960 & 0.3 & 0.18 & 0.966 & 0.2 & 0.17 & 0.961 & 0.3 & 0.12 & 0.953 \\
P50 & 0.1 & 0.09 & 0.965 & 0.2 & 0.13 & 0.970 & 0.2 & 0.15 & 0.962 & 0.2 & 0.08 & 0.967 \\
P25 & 0.2 & 0.20 & 0.953 & 0.3 & 0.17 & 0.963 & 0.3 & 0.24 & 0.950 & 0.1 & 0.11 & 0.955 \\
P10 & 0.3 & 0.15 & 0.955 & 0.1 & 0.23 & 0.955 & 0.1 & 0.18 & 0.957 & 0.2 & 0.09 & 0.960 \\
\hline
\end{tabular}

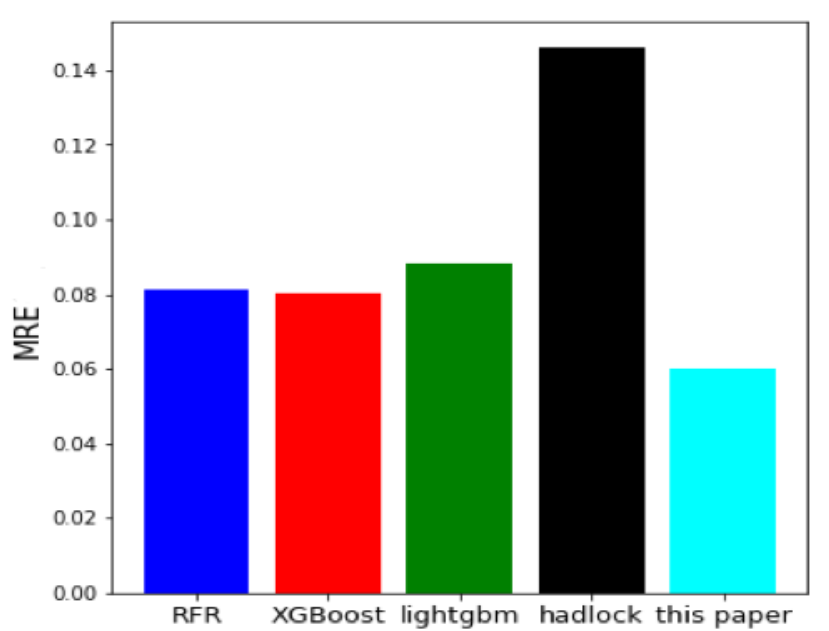

Figure 3: Mean relative error of different models.

Table 3: IoU based on different machine learning algorithms.

\begin{tabular}{lr}
\hline Algorithms & Intersection-over-Union \\
\hline Random forest & 0.607 \\
XGBoost & 0.623 \\
LightGBM & 0.610 \\
Our model & 0.650 \\
\hline
\end{tabular}

and the random forest models, respectively, and the ordinate expresses by the predicted fetal weight range. From the graph results, after optimising the multi-model parameters based on the genetic algorithm, the advantages of each model can be effectively utilised, so that the fetal weight prediction interval is closer to the fetal weight range of the ultrasound examination.

\section{Analysis of Fetal Growth Change}

The fetal growth curve is an important index of the fetal health status, which can provide a basis for early diagnosis and the prevention of fetal abnormalities. At the same time, pregnant women can observe the trend of fetal weight changes in each gestational week, including the average fetal weight curve, the 10th percentile curve and the 90th percentile curve. Therefore, based on the characteristic parameters of pregnant women and the fitted ultrasound charac-
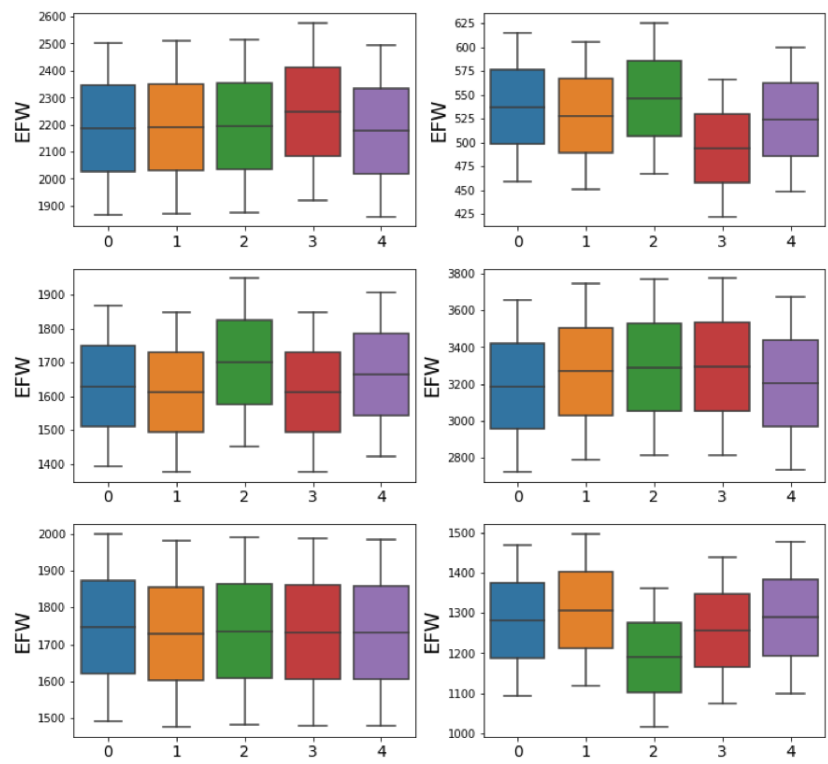

Figure 4: Mean relative error of different models.

teristic parameters, this study uses the ensemble model to predict the fetal weight at the current moment and to timely understand the trend of fetal growth.

When comparing with the 10th and 90th percentiles of China's fetal growth standard curve (Lei and Wen 1998), if the curve is lower than the 10th percentile, the fetus is small for its gestational age (SGA), and conversely, when the curve is greater than the 90th percentile, the fetus is large for its gestational age (LGA). A sample was randomly selected from the testing set, and the fetal weight is predicted by the ensemble model proposed in this study. The experimental results are shown in Figure 5.

\section{Discussion}

To verify the superiority of the model, the ensemble model is used to predict the birth weight of the fetus. From the testing sets, 527 samples have a record of an ultrasound examination within one week prior to delivery. In addition, it is more objective to evaluate the accuracy of different algorithms, this study uses another criterion, that is, the error between the predicted and actual fetal weight is within $\pm 250 \mathrm{~g}$, and the prediction is considered to be accurate (Jain, Duin, and 


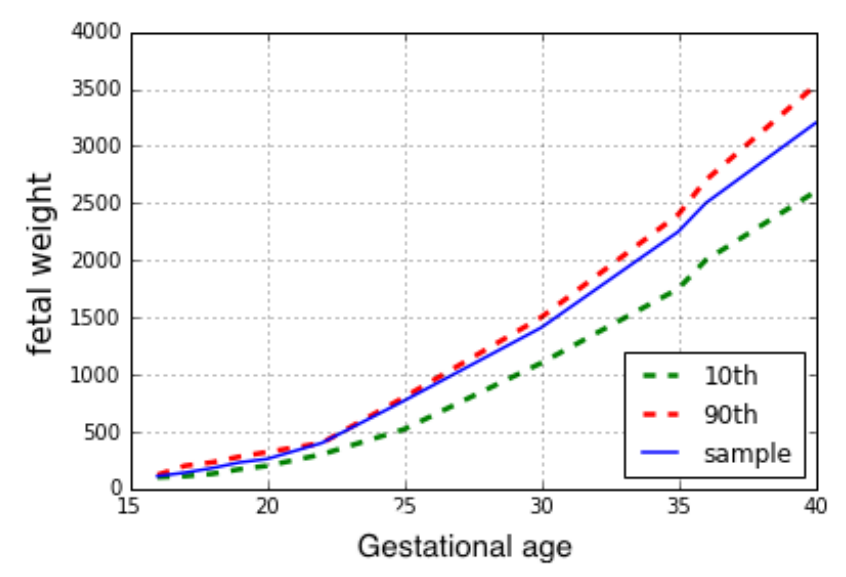

Figure 5: Fetal growth prediction curve.

Mao 2000). Therefore, different algorithms are used to predict fetal birth weight. The experimental results are shown in Table 4.

Table 4: Different methods that predict the fetal birth weight.

\begin{tabular}{lrr}
\hline Parameters & MRE (\%) & Accuracy (\%) \\
\hline Hadlock (Hadlock 1990) & 10.2 & 52.3 \\
GA-BP (Zhu et al. 2018) & 7.5 & 63.1 \\
Random forest & 8.3 & 60.0 \\
XGBoost & 8.2 & 62.1 \\
LightGBM & 8.4 & 59.4 \\
Proposed ensemble model & 7.0 & 64.3 \\
\hline
\end{tabular}

Table 4 shows that the ensemble model proposed in this paper predicts the fetal birth weight and has a certain degree of improvement in the MRE and accuracy compared with the single machine learning algorithm model and the multiparameter method. The MRE is reduced by approximately $3 \%$, and the accuracy is improved by approximately $12 \%$.

\section{Conclusion}

This paper proposed a novel approach to estimating fetal weight using ensemble machine learning algorithms. The cubic spline function has been used to fit the functional relationship between the BPD, AC, HC, and FL and the gestational age based on the health records of pregnant women. An ensemble machine learning model has been proposed based on the genetic algorithm with parallel optimisation of multiple parameters to predict the fetal weight at varying gestational age. We have also evaluated the applicability of the ensemble model for the domain of on real datasets. Comparing with the traditional ultrasound-based estimation methods, it obtains the EFW more accurately and efficiently. Next, there are further clinical tests in different hospitals, and software for both home and hospital applications are under development and soon to be deployed. Estimation of fetal birth weight among twins is another direction of future research.

\section{Acknowledgements}

This work is supported by the National Natural Science Foundation of China (No. 61272328) and 2017 Science and Technology Plan Project of Shenzhen, China (KJYY20170721163528274).

\section{References}

Breiman, L. 2001. Random Forests. Machine Learning 45(1):5-32. Chen, T., and Guestrin, C. 2006. XGBoost: A Scalable Tree Boosting System. In KDD 2016, 785-794.

Conde-Agudelo, A.; Papageorghiou, A. T.; Kennedy, S. H.; and Villar, J. 2013. Novel biomarkers for predicting intrauterine growth restriction: a systematic review and meta-analysis. BJOG 120(6):681-694.

de Onis, M. 2006. WHO Child Growth Standards based on length/height, weight and age. Acta Paediatrica 95(S450): 76-85.

Dietterich, T. G. 2000. An Experimental Comparison of Three Methods for Constructing Ensembles of Decision Trees: Bagging, Boosting, and Randomization. Machine Learning 40(2):139-157.

Ewigman, B. G.; Crane, J. P.; and Frigoletto, F. D. 1993. Effect of Prenatal Ultrasound Screening on Perinatal Outcome. The New England Journal of Medicine 329(12):821-827.

Hadlock, F. P. 1990. Sonographic estimation of fetal age and weight. Radiologic Clinics of North America 28(1):39-50.

Jain, A. K.; Duin, R. P. W.; and Mao, J. 2000. Statistical pattern recognition: a review. IEEE T. PAMI 22(1):4-37.

Ke, G.; Meng, Q.; and Finley, T. 2017. LightGBM: A Highly Efficient Gradient Boosting Decision Tree. In NIPS 2017.

Lei, H., and Wen, S. W. 1998. Ultrasonographic examination of intrauterine growth for multiple fetal dimensions in a chinese population. AJOG 178(5):916-921.

Miller, S. L., and Huppi, P. S. 2016. The consequences of fetal growth restriction on brain structure and neurodevelopmental outcome. Journal of Physiology 594(4):807-823.

Naimi, A. I.; Platt, R. W.; and Larkin, J. C. 2018. Machine Learning for Fetal Growth Prediction. Epidemiology 29(2): 290-298.

Podda, M.; Bacciu, D.; and Micheli, A. 2018. A machine learning approach to estimating preterm infants survival: development of the Preterm Infants Survival Assessment (PISA) predictor. Scientific Reports 8(13743):1-9.

Pressman, E. K.; Bienstock, J. L.; Blakemore, K. J.; Martin, S. A.; and Callan, N. A. 2000. Prediction of birth weight by ultrasound in the third trimester. Obstetrics \& Gynecology 95(4):502-506.

Schapire, R. E. 1990. The strength of weak learnability. Machine Learning 5(2):197-227.

Wanyonyi, S. Z., and Mutiso, S. K. 2018. Monitoring fetal growth in settings with limited ultrasound access. Best $P \& R$ Clinical $O b$ stetrics \& Gynaecology 49:29-36.

Zhu, H.; Tao, J.; Yu, K.; and et al. 2018. Fetal Weight Prediction Analysis Based on GA-BP Neural Networks. Computer Systems \& Applications 27(3):162-167. 